through the permissions, Mattioli says.

Spurred on by the devastating 2010 Haiti quake, the network aims to fill out the currently patchy picture of the region's geological turmoil. Off the Pacific coast of Costa Rica, for instance, a COCONet station on the isolated Isla del Coco is the only GPS station continuously tracking the Cocos plate as it dives beneath the Caribbean plate. The data show that the two plates are converging at a rate of 78 millimetres per year. Such information will help geophysicists to reconcile their ideas about plate motion in the region, says Marino Protti, a geologist at the National University of Costa Rica in Heredia, who presented his findings in Cancún.

Other COCONet stations dot Costa Rica's Nicoya Peninsula, one of the few places on land that lie directly above a subduction zone. In
September 2012, Nicoya shuddered from a magnitude-7.6 earthquake. "Having the network helped us see that it was not a whole-plate event," Protti says. He thinks that remaining stress in the plate could trigger another large tremor.

Besides tracking movements in Earth's crust, the GPS data allow atmospheric scientists to measure how much water vapour sits in the air between each station and the GPS satellites, based on the delay in the signal's travel time. That information is a rare, real-time glimpse of how much water is available to power hurricanes, says meteorologist John Braun of the University Corporation for Atmospheric Research in Boulder.

COCONet is even yielding some unexpected societal benefits. In the Dominican Republic, land surveyors are eagerly using the nation's nine stations to comply with a real-estate law that requires precise surveying of parcels of land for sale, says Alexander Holsteinson of the National University of Pedro Henríquez Ureña in Santo Domingo.

Yet funding for COCONet runs out in 2015, and no one knows what will happen then. Each country will have to find the money to keep its stations operating, says Guoquan Wang, a geophysicist at the University of Houston, Texas, and a COCONet member who developed a GPS network for Puerto Rico and the Virgin Islands. That means that host nations will have to find value in supporting the project, says Héctor Mora Páez, who heads the GPS network for the Colombian Geological Survey in Bogota. "You have to make the local people feel involved." -
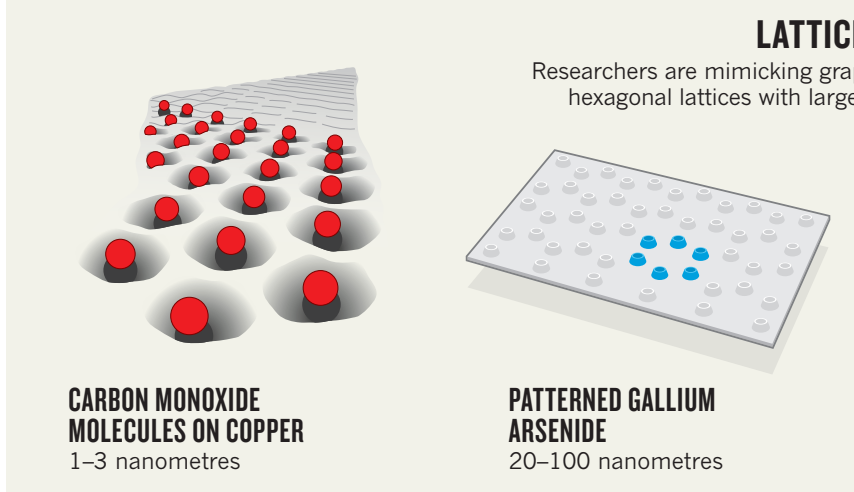

LATTICE LOOKALIKES

20-100 nanometres

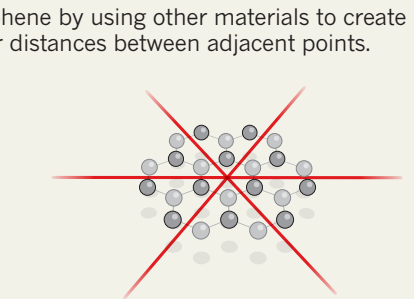

POTASSIUM ATOMS

TRAPPED BY LASERS

500 nanometres

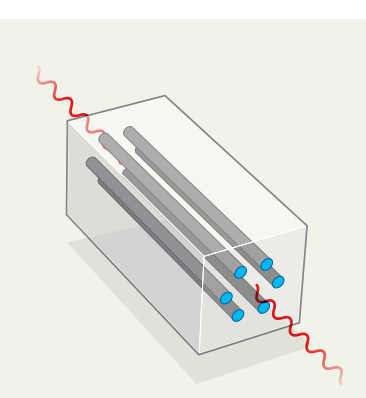

MICROWAVES BETWEEN CERAMIC CYLINDERS

15 millimetres

\title{
Graphene knock-offs probe ultrafast electronics
}

\section{Honeycomb lattices in different materials enable experiments impossible in the real thing.}

\section{BY EUGENIE SAMUEL REICH}

$\mathrm{I}$ f imitation is the sincerest form of flattery, then graphene is basking in admiration. Several labs are recreating the six-fold geometry of the carbon-based material with a range of building blocks, hoping to match - if not surpass - graphene's fascinating properties.

The approaches span a range of length scales, from nanometres to millimetres (see 'Lattice lookalikes'), and take advantage of a variety of construction techniques, including potassium atoms trapped by laser beams, hexagons etched on a gallium arsenide surface and microwaves pumped into a honeycomb arrangement of ceramic cylinders. The research is mostly motivated by curiosity, and few think that any of the 'artificial graphenes' will be used in applications. But the new materials show that hexagonal patterns are worth investigating. "It's clear the physics in electrons in graphene is very attractive," says Eros Mariani, a theoretical physicist at the University of Exeter, UK.

Graphene has unusual electronic properties as a result of its Dirac points, positions in its single-sheet honeycomb lattice of carbon atoms at which the energy bands of electrons from adjacent atoms link up. These allow electrons in the bands to speed across the material, like cars changing roads at major intersections instead of getting stuck on one jammed road. Measurements reported ${ }^{1,2}$ in 2005 confirmed that electrons in graphene move so fast that they seem to have no mass, and are, in effect,

moving at the speed of light. That fuelled hopes that graphene could be used to make ultrafast electronics; it also set the stage for the discovery of exotic quantum-mechanical effects.

In 2010, physicist Hari Manoharan, an expert in atomic manipulation at Stanford University in California, stumbled on the first graphene mimic. Manoharan and his team were using a scanning tunnelling microscope to arrange various molecules on a copper surface when they were struck by a hexagonal pattern. It looked

$\rightarrow$ NATURE.COM For more on the properties of graphene, visit: go.nature.com/hm $41 \mathrm{sm}$ uncannily like graphene but was eight times larger in scale. "We were thinking, why don't we see if it has graphene's properties?" says Manoharan. 
He and his colleagues soon settled on carbon monoxide as their molecule of choice, and in 2012, they published evidence ${ }^{3}$ that carbon monoxide molecules arranged in a honeycomb pattern do have Dirac points. And in the same year, Tilman Esslinger, a physicist at the Swiss Federal Institute of Technology in Zurich, showed that a gas of ultra-cold potassium atoms trapped $^{4}$ in a hexagonal lattice by criss-crossing laser beams also sustains faster-than-light conduction - but of atoms, rather than electrons.

Both groups then performed tricks that are currently impossible in real graphene. Manoharan and his colleagues deposited their molecules in a slightly warped pattern, which caused the energy levels of the electrons in the lattice to split as if they had been exposed to a magnetic field stronger than any that could be applied in a laboratory. "You can trick electrons into thinking they're in a 60-tesla magnetic field," says Manoharan.

Esslinger and his team also distorted their lattice, fading out one of the laser beams to shift the structure from a hexagonal to a dimeric one, built of pairs of atoms ${ }^{4}$. That prompted the precious Dirac points to merge and annihilate. With the intersections gone, the atoms got trapped in lower energy bands and were less free to move, confirming that the hexagonal laser lattice really had been mimicking the electronic structure of graphene.

In January, Fabrice Mortessagne, a physicist at the University of Nice Sophia Antipolis in France, and his colleagues demonstrated another feat of distortion ${ }^{5}$. His group assembled hexagonal arrangements of millimetrescale ceramic cylinders between two metal plates. They then pumped microwaves into the lattice. The waves became trapped there, and their movement mimicked the path of electrons in graphene. When the researchers squeezed the lattice in one direction, they found that the microwaves were trapped unexpectedly along the edges of the structure, which challenges current theoretical descriptions of Dirac materials. The group suggests that similarly unexpected energy states will be observed for electrons in graphene once lab techniques have been developed to compress the material in one direction without breaking it.
Philip Kim, a physicist at Columbia University in New York who was involved in the 2005 measurements that uncovered graphene's unusual electronic properties, finds artificial graphenes exciting because they can be engineered to show effects expected in real graphene. But simply replicating graphene's properties doesn't grip everyone. "To mimic graphene sounds pretty boring scientifically and a waste of time and resources," says Antonio Castro Neto, director of the Graphene Research Centre at the National University of Singapore. "The objective of creating these artificial graphene-like lattices is to produce new systems that have properties that graphene does not have."

For example, he has predicted ${ }^{6}$ that etching a honeycomb structure onto a semiconductor such as gallium arsenide could turn it into a topological insulator - a much-sought material that insulates in its interior but conducts superbly well on its surface, where exotic particles called Majorana fermions might emerge. These particles, which have been tentatively observed in one-dimensional nanowires, have a unique ability to encode quantum information by swapping locations with each other (see Nature 483, 132; 2012). Hexagonal gallium arsenide lattices have been made on large scales, but Castro Neto says that current methods of nanolithography should enable researchers to etch them at scales of 20 nanometres or less, fine enough to see the Majoranas.

Although it will be a long time before artificial graphenes can be used in quantum computing, Castro Neto doesn't rule out a pay-off for them. "I am always surprised that people find applications for things I thought would never be useful," he says.

1. Zhang, Y., Tan, Y.-W., Stormer, H. L. \& Kim, P. Nature 438, 201-204 (2005).

2. Novoselov, K. S. et al. Nature 438, 197-200 (2005).

3. Gomes, K. K., Mar, W., Ko, W., Guinea, F. \&

Manoharan, H. C. Nature 483, 306-310 (2012).

4. Tarruell, L., Greif, D., Uehlinger, T., Jotzu, G. \& Esslinger, T. Nature 483, 302-305 (2012).

5. Bellec, M., Kuhl, U., Montambaux, G. \& Mortessagne, F. Phys. Rev. Lett. 110, 033902 (2013).

6. Sushkov, O. P. \& Castro Neto, A. H. Preprint at http://arxiv.org/abs/1210.8186 (2013).

priming effect reported by Ap Dijksterhuis. We note that other researchers have observed Dijksterhuis's intelligence-priming effect. - Contrary to the story, Dijksterhuis has stated that he will provide a protocol to assist in replicating results in the field. He had not, however, provided such a protocol at the time that the story was published.

- As the story stated, there is no suggestion of fraud in Dijksterhuis's research, and we are happy to emphasize that there is no suggestion that he has been involved in any misconduct. 